\title{
Research and Design of RFID-based Equipment Incident Management System for Industry $\mathbf{4 . 0}$
}

\author{
Jiqing $\mathrm{CaO}^{1, \text { a }}$, Shuhai Zhang ${ }^{2, b}$ \\ ${ }^{1}$ Department of Information Engineering, Suzhou Industrial Park Institute of Service Outsourcing \\ ${ }^{22}$ Department of IT Service, Bosch Automotive Products (Suzhou) Co., Ltd. \\ aemail:grinner@tom.com, bemail:zhangsh @tom.com
}

Keywords: RFID; Industry 4.0; Incident Management; ITIL; Cloud Computing;

\begin{abstract}
RFID technology is the key technologies in the field of IOT, this paper studies the application scenarios of RFID technology in Industry 4.0 incident management, designs an architecture of the Equipment Incident Management System based on cloud platform, improves the traditional ITIL-based Incident Management Process and designs a new Incident Management Process based on technologies of RFID, intelligent positioning and Artificial Intelligence. The system implements the functions of Equipment Management, Personnel Management, Intelligent Distribution Management, Location Management, Visualization Management, Incident Knowledge Base Management and the process of Data Collection, Analysis and Application of Decision-making, which can greatly enhance the effectiveness of Industry 4.0 System incident management. Currently, the system has been effectively used in a company's equipment management system.
\end{abstract}

\section{Introduction}

There are thousands of instruments, meters and equipments installed in vary of integrated systems in Industry 4.0 system. The incident management for these equipments will directly affect the stable operation of the system. Efficient Operation and Maintenance mode is, when there is a fault or warning of the equipment, technical personnel will be able to understand the properties of the failed device, location, and operation and maintenance history and other information before reaching the fault site, so as to make timely targeted anticipation, and the system has get ready for the appropriate spare parts or maintenance program; and the status information of the equipment operation and maintenance can be shared to the system after the completion of the maintenance, so as to facilitate the latter part of the operation and maintenance. [1]

With the IoT (Internet of Things) feature technology of RFID (Radio Frequency IDentification), this paper designs the architecture of the Incident Management System, establishes a new automated Incident Management Process based on the traditional ITIL-based Incident Management Process, which achieves the automation, visualization and location management of the Incident Management, and solve the problems of the big workload, time-consuming, and high costs of the Incident Management in Industry 4.0.

\section{RFID Technology}

RFID technology, also known as electronic tags, is a communication technology. It can identify specific targets and read and write data, without the need for the establishment of contact between the mechanical or optical recognition systems with a specific target through radio signals. RFID technology has advantages of waterproof, anti-magnetic, high temperature resistant, large read range, fast batch identification, small size, large data storage capacity, rewritable storage information, etc., and it adapts to the complex environment of Industry 4.0 system, the application of RFID will bring revolutionary change to Industry 4.0.

RFID system consists of three parts, the TAG, the READER and ANTENNA. RFID READER works by sending out a certain frequency of the RF signal through an ANTENNA, the label obtains 
energy through the induced current when enters the magnetic field, the information of its own coding sent out are read and decoded by the READER and sent to the computer system for processing.

The use of RFID technology can build bridges between physical space and information space, realize the binding between a physical space object and information space object, and provide rich information for the Internet of Things and people. RFID data collection needs no human involvement, it reads with high speed and enables batch read. Coordinated with other technologies, it can effectively improve the capabilities of equipment management, storage management, location management and visualization management. [2]

\section{Traditional ITIL-based Incident Management Process}

ITIL (IT Infrastructure Library) is currently the international de facto standard for IT operations. ITIL describe the "incident" as "Unexpected IT service disruption or Degradation of IT service quality. Configure Item has not been an impact on the service failure is also an incident, such as a node in clusters goes down.” The Incident Management Process of ITIL is a process for handling all incidents that may be reported from varies of ways and has been known thousands of businesses successfully managed IT system failures in the past decade.

ITIL Incident Management Process comprises the steps of: Incident Source, Incident Confirmation, Incident Records, Incident Classification, Incident Priority, Initial Diagnosis, Escalation, Judgment, Investigation and Diagnosis, Resolution and Recovery, Incident Close [3]. This process contains the activities of up to eleven steps and involves multiple roles and functions such as support, technology, quality and business etc, which will cause quite a few time delays and reduce the efficiency of the incident treatment. In the same time, it has still not considered the impact of new technologies such as RFID and virtual resources on the process. [4]

\section{RFID-based Incident Management System Design}

\section{Design Principal and Purpose}

Considering the characteristics of Industry 4.0, the design principles for the Incident Management System are: to follow ITIL standards, use RFID, Cloud Computing, Virtual Reality, Intelligent Positioning and Automation technologies to realize the Cloud-based, automation and visualization for the Industry 4.0 Incident Management.

Specific objectives are as follows: to use RFID technology to realize the functions of automatic identification of the equipment, automatic collection of the equipment information, automatic alarm of the failures, location management of the equipment and personnel; to use the cloud-base Incident Management System to realize the automatic inspection and incident management for the three different directions of the Industry 4.0 system, i.e., Horizontal Integration Systems, Vertical Integration System and End to End Integration System.

\section{Design of the Incident Management System}

Based on the above design principles and objectives, the designed Incident Management System architecture (see Figure 1) is established on the cloud platform, it is divided into three Layers, they are Data Acquisition Layer, that is layer of IaaS (Infrastructure as a Service), Platform Management Layer, that is PaaS (Platform as a Service,), and Data Services Layer, that is the layer of SaaS (Software as a Service,). Data Acquisition Layer is responsible for the data collection for the cross-system, cross-regional infrastructure and production equipment through the wireless communication technologies of RFID, 4G, WIFI and ZigBee and technology of ESB (Enterprise Service Bus) middleware [5]. Platform Management Layer provides the core functions of RFID middleware, Cloud Computing, Virtual Reality, Visualization and Automation Engines and etc; Data Services Layer completes the core functions of Incident Management, it is the unified interface opened to the Industry 4.0 users and other systems. 


\begin{tabular}{|c|c|}
\hline $\begin{array}{l}\text { Data Service } \\
\text { Layer } \\
(\text { SaaS })\end{array}$ & $\begin{array}{l}\text { Mgt of Incident Process, Equipment, } \\
\text { Personnel, Position, Visualization } \\
\text { Incident Knowledge Base }\end{array}$ \\
\hline $\begin{array}{l}\text { Platform Mgt } \\
\text { Layer } \\
\text { (PaaS) }\end{array}$ & $\begin{array}{c}\text { Configuration Mgt Database } \\
\text { Platform of RFID, Cloud Computing, } \\
\text { Position, Automation Engine, Visualization }\end{array}$ \\
\hline $\begin{array}{l}\text { Data Acq. Layer } \\
\text { (IaaS ) }\end{array}$ & $\begin{array}{l}\text { RFID System, ESB, Equipments, Sensors } \\
\text { 4G, WIFI, ZigBee }\end{array}$ \\
\hline
\end{tabular}

Fig.1. RFID-based Incident Management System Architecture

The functions provided by the unified interface of the Incident Management System include Incident Process Management, Equipment Management, Personnel Management, Visualization Management and Incident Knowledge Base, introduced as followings:

\section{Incident Process Management Module}

The process achieves the functions of automated handling for the Failures and Alarms. Considering the impact of RFID and other new technologies for Incident Management Process, the paper improves the traditional ITIL-based Incident Management Process (see Figure 2). The new process is divided into five activities, namely, Incident Monitoring, Incident Pretreatment, Spare Parts Management, Intelligent Distribution and On-site Installation.

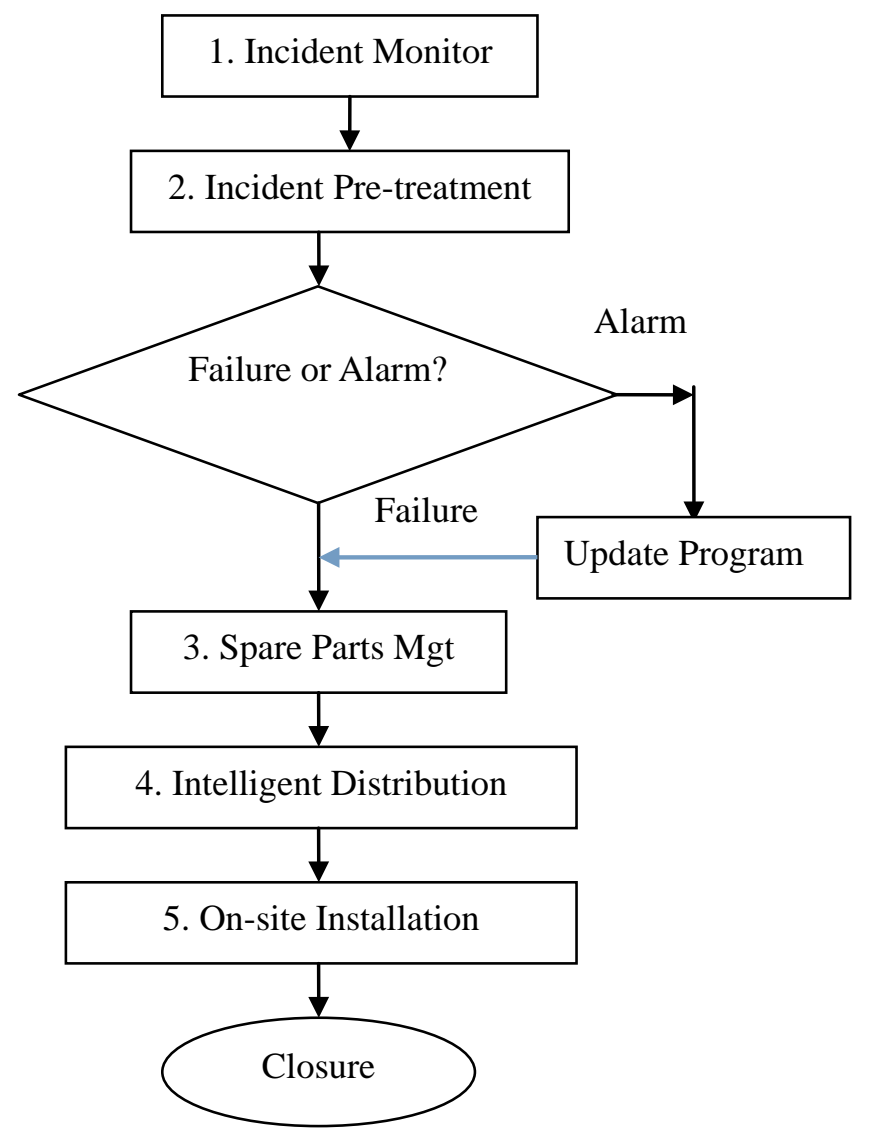

(1) Incident Monitoring

Fig.2. Updated RFID-based Incident Management Process

In this Module, the system reports failures or warnings from Data Acquisition Layer through RFID and other technologies. Combined with sensor system by the upper limit of temperature, humidity and vibration amplitude, etc. set in advance, the system can notify the failure and alarm 
information to the Incident Management System by the wireless communication, and push through RFID handhelds and other ways to notify maintenance personnel.

(2) Incident Pretreatment

This Module is responsible for recording, classifying and judgment of the failures or warnings reported from the Incident Monitoring Module which has integrated the multi-steps of activities of the traditional ITIL-based Incident Management Process.

If it is a failure, the process will go to the activity of Device Management; else the system will update the Incident Maintenance Program including the information of spare parts requirements, warehousing, distribution and task owner etc for the corresponding equipment and follow the same process as the failure before the warning is closed.

(3) Spare Parts Management

This Module is based on the Equipment Management Module of the whole Incident Management System and is responsible for the management of the replaceable equipments for the corresponding incident reported from RFID. This module can quickly and accurately calculate the number and locate the position of the required spare parts and alarm when they are insufficient.

(4) Intelligent Distribution

This function associates RFID technology and location monitoring of the vehicle, equipment and spare parts, and intelligently delivers the spare parts to the installation site. Through RFID positioning technology, the systems automatically assign the task to the adjacent maintenance staff and send the information of installation location, installation methods, attributes, manufacturers, technical support and etc to the maintenance personnel.

(5) On-site Installation

This module is responsible for the installation, testing and validation of the spare parts on site. When the spare parts are transported to the designated site, the operation and maintenance personnel or artificial intelligence robot will implement the installation of spare parts as the circumstances and conditions. When repairing or replacing the targeted equipment, the Incident Management System can push information of the related spare parts to RFID handheld terminal. After installation is completed, the operation and maintenance personnel can update the information like equipment status rack room, IP, installation date and other information in the Incident Management System via RFID handheld terminal to ensure the accuracy of the data.

\section{Equipment Management Module}

Features of this module include Registration of RFID Equipment, Monitoring Management, Alarm Management and Spare Parts Management and etc. This module implements the real-time monitoring of the status of production equipment and realizes timely repair of the faulty equipment. It improves the efficiency of the use and maintenance of the production equipment.

(A) Registration of RFID Equipment: This sub-module designs the RFID tags which meet the practical requirements and corresponds to the equipment and maintenance personnel, maintenance the corresponding relationship between the locations and the READERs, and labels and items. The contents of the label include information of the equipment attributes, installation, previous failure causes and associated processing and other information. [6]

(B) Monitoring Management: In routine maintenance, the system maintenance personnel obtain conventional operation and maintenance information and add and update the latest maintenance information via the RFID READER. When the equipment reaches scrap processing, the system will automatically alert staff to do scrap processing, thus implement the whole life cycle management of the equipment.

(C) Alarm Management: The way to repair after the failure occurs will not only take longer, but also cause the unnecessary economic losses of the enterprise. There are many incident prediction methods to learn from, including Neural Network Algorithm, Kalman Filtering Method etc., advantages and disadvantages of each method and scope of application is different. This function creates an alarm policy repository, sets alarm thresholds for each collected state data by sensors. Whenever data collected from the equipments, it calls the alarm threshold engine to do the check and raises the alarm if it exceeds the threshold. [7] 
(D) Spare Parts Management

Based on RFID technology, the spare parts management achieves the automation of data collection and storage, conducts a comprehensive control and management for the storage, a library and inventory and other operations for the spare parts. When the number of spare parts inventory is insufficient, it is possible to alert early, and automatically generate purchase orders to replenish ullage. The module can reduce the work intensity of the maintenance personnel, enhance the level of automation and visualization of the spare parts management, and improve its management efficiency and effectiveness. [8]

\section{Personnel Management Module}

This module designs the RFID tag to meet the practical requirements and establishes the association between the personnel and the equipment. The contents of the tag include the name of the operation and maintenance staff, the owned equipments, region and time range and etc. When the equipment fails, the appropriate operation and maintenance personnel will be accurately assigned to make a timely process. Combined with technologies of visualization and positioning, the system also realizes the visual management of the positions of the people and the equipment. [9]

\section{Position Management Module}

This module implements intelligent positioning and tracking for the equipment, spare parts, delivery vehicles, and operation and maintenance personnel to make a timely response and handling at any time when failure occurs. When it needs to locate the relevant equipment, the user just clicks to find the equipment by selecting the RFID ID number in the system. On one hand, the system can display specific location of the equipment in the virtual environment through visualization technology; on the other hand, the system can quickly guide the personnel to locate and find items by sending commands to the attached RFID tags of the equipment to trigger the tag buzzer and LED lights. [10][11]

\section{Visualization Management Module}

Currently, the combination of RFID, Wireless Sensor Networks and Virtual Reality technology and other technologies to achieve Visualization has matured. Three-dimensional Virtual Reality technology can virtually display the location and status of the factory buildings, the internal passageway of the workshop and production equipments.Failure, alarm and key target trajectory information can be displayed superimposed in virtual systems, so that it provides a platform for integrated information display and interaction. [12]

\section{Incident Knowledge Base Module}

The module maintains knowledge of field failure analysis, solutions and reasons summaries. The maintenance personnel can query the basic information and methods of troubleshooting, anytime, anywhere via the mobile terminal, so that the general staff can solve the general failures. Thus, operation and maintenance of data, information, processes, methods etc. are preserved, which helps to train the operation and maintenance team.

\section{Benefit Analysis}

Company A is an IC factory, which has 20 production lines, with the equipment and instruments required for the management of nearly one thousand. Before adopting RFID to manage the operation and maintenance of the equipment, the plant's average processing time for a single device incident is about 1 hour (including time of incident detection, time of the patrol personnel arrive onsite, data query time, time to prepare the spare part, time of troubleshooting and testing, etc.), the average production line calibration period is about four months, OEE (Overall equipment Effectiveness, OEE) is about $80 \%$, the average monthly cost in equipment maintenance time is about 500 hours. The time spent on the maintenance of the equipment takes a lot of maintenance costs. After using this RFID Incident Management System in Company A, the incident management time for each equipment has reduced by an average of 40 minutes, the OEE has increased to $90 \%$, the average monthly time spent on equipment maintenance has reduced 400 hours, and the annually saved costs of the labor on maintenance have been 4.8 million YUAN after using the RFID-based Incident Management System. 


\section{Summary}

The RFID Incident Management System enables functions of incident monitoring, early warning, positioning, automation and visualization management etc for the equipment. The updated ITIL-based Incident Management Process can greatly enhance the work efficiency of operation and maintenance of the equipment and the inspection personnel. The system has been put into use in an IC factory. It uses RFID technology to manage the equipment and the troubleshooting process, and change the traditional mode of manual inventory of the equipment and processing of the incidents, which liberates the labor and achieves considerable economic benefits.

\section{Acknowledgement}

In this paper, the research was sponsored by the Service Outsourcing Foundation of Suzhou Institute Park Service Outsourcing Institute (Project No. ky-xjy02).

\section{References}

[1] Xia Huayu, Wang Tao, Jiang Jia. Application of Mobile Technology in the Operation and Maintenance of the Metering Device [J]. Engineering and Technology: Digest Edition, 2015, (66)

[2] Yin Zhouping, Tao Bo.. Intelligent Manufacturing and RFID Technology[J].Aeronautical Manufacturing Technology 2014, (3)

[3] Service_operation.ITIL Version 3 (2011):72-86

[4] Chen Chunhua, Li Jun.Liang Huan,Yao Wensheng. IT Service Management Implementation Strategy of Resource Pool Environment[J]. Telecommunications Science, 2012(9) :144-146

[5] DI Jian [1] XIAO Jun [1] WANG Chunxin [2] YANG Youpeng [2] Design of IoT comprehensive service platform based on enterprise service bus[J] .Journal of Computer Applications

[6] Xu Dan, He Zhiguo. Fixed Assets Management System Based on RFID Technology[J]. Computer \& Digital Engineering, 2010, (12)

[7] Wang Yan. Design and Implementation of the Pre-maintenance Incident Diagnosis System Based on RFID[J]. Engineer Technology, 2015, (12)

[8] Zhang Kangyi, Xue Jijun, Wang Rui. Management of Spare Parts Based on RFID [J]. Ordnance Industry Automation, 2015-0534(5)

[9] Sun Dihua, Wang Lei, Song Xiaoxiao, Yang Fan. RFID-based Automatic Worker Identification for Lean Production [J]. Modern Manufacturing Engineering, 2010, (12)

[10] Yan Baozhong, Zhang Shuai. Design and Implementation of the Indoor Personnel Positioning System Based on RFID [J]. Applied Science and Technology, 2011, (11)

[11] Liao Xingxing, Jin Gang, Chen Xiaoguang. A RFID Technology Based Visualization Method of Equipment Location [J]. Modern Electronics Technique, 2016, (6)

[12] Chen Guojing, Duan Qichang.The in-transit Visibility System Based on RFID and Wireless Sensor Network[J]. Industrial Instrumentation \& Automation, 2007, (1) 